\title{
Applicability of the Wong formula for fusion cross sections from light to heavy systems
}

\author{
N. W. Lwin, ${ }^{1}$ N. N. Htike, ${ }^{1}$ and K. Hagino ${ }^{2,3,4}$ \\ 1 Department of Physics, Mandalay University, Mandalay, Myanmar \\ 2 Department of Physics, Tohoku University, Sendai 980-8578, Japan \\ ${ }^{3}$ Research Center for Electron Photon Science, Tohoku University, 1-2-1 Mikamine, Sendai 982-0826, Japan \\ ${ }^{4}$ National Astronomical Observatory of Japan, 2-21-1 Osawa, Mitaka, Tokyo 181-8588, Japan
}

\begin{abstract}
We discuss the applicability of the Wong formula for fusion cross sections in a single-channel problem. To this end, we carry out a systematic study and compare the approximate fusion cross sections with the exact results in a wide mass region of reaction systems. We show that the deviation of the approximate results from the exact cross sections is large for light systems, even though the Wong formula provides a reasonable approximation for heavy systems. We also discuss the energy dependence of the deviation, and show that for a given projectile nucleus the critical energy, at which the deviation exceeds $5 \%$ of the exact cross sections, increases as a function of the mass number of the target nucleus.
\end{abstract}

\section{INTRODUCTION}

The nuclear fusion reaction is defined as a reaction to form a compound nucleus. It plays an essential role in several phenomena in physics, such as the energy production in stars, nucleosynthesis, and a synthesis of superheavy elements [1 $[4]$. Theoretically, the simplest approach to estimate fusion cross sections is to use the potential model, in which one assumes a spherical potential between the two colliding nuclei. In this model, the colliding nuclei are assumed to be spherical and inert, and fusion is simulated as an absorption of flux inside the Coulomb barrier. Fusion cross sections are then obtained by adding the penetrability of the Coulomb barrier for each partial wave.

Approximating the Coulomb barrier with a parabolic function, Wong has derived a compact expression for fusion cross sections [5]. The resultant formula, referred to as the Wong formula, has been widely used in the investigation of heavy-ion fusion reactions. For instance, the formula has been used to provide reference cross sections in order to discuss channel coupling effects. The formula has also been used recently in order to analyze fusion barrier distributions using a method based on the Bayesian spectral deconvolution [6]. Moreover, the correction to the Wong formula has been discussed in order to discuss the oscillatory behavior of fusion cross sections in symmetric and nearly symmetric light systems [7, 8].

The original Wong formula tends to overestimate fusion cross sections in light systems. In order to cure this problem, the Wong formula has been generalized in Ref. 8] by introducing an energy dependence to the barrier parameters. Even though the generalized Wong formula has been shown to reproduce well the exact results, it has yet to clarify from which system and from which energy the generalization becomes important. The aim of this paper is to perform a systematic study and address this question. To this end, we compare fusion cross sections obtained with the Wong formula with the exact results from light to heavy systems. We shall discuss the deviation from the exact cross sections as a function of the mass number of the target nucleus for a fixed projectile nucleus.

The paper is organized as follows. In the next section, we will briefly present the formulation of the potential model for fusion reactions, and introduce the Wong formula. In Sec. III, we will apply the Wong formula to a multitude of systems and discuss its applicability. We will also introduce the critical energy, at which the deviation exceeds a certain fraction of the exact cross sections. We will finally summarize the paper in Sec. IV.

\section{THE WONG FORMULA FOR FUSION CROSS SECTIONS}

In the potential model, fusion cross sections are computed from a numerical solution of the radial Schrödinger equation,

$$
\left[-\frac{\hbar^{2}}{2 \mu} \frac{d^{2}}{d r^{2}}+V_{0}(r)+\frac{l(l+1) \hbar^{2}}{2 \mu r^{2}}-E\right] u_{l}(r)=0
$$

where $\mu$ is the reduced mass, $V_{0}(r)$ is an inter-nucleus potential (that is, a sum of a nuclear and the Coulomb potentials), $l$ is the relative angular momentum between the colliding nuclei, and $E$ is the incident energy in the center of mass frame. With the incoming wave boundary condition, this equation is solved with boundary conditions of [2, 9]

$$
\begin{aligned}
u_{l}(r) & \sim \sqrt{\frac{k}{k_{l}(r)}} T_{l} \exp \left(-i \int_{r_{\min }}^{r} k_{l}\left(r^{\prime}\right) d r^{\prime}\right), \quad r \leq r_{\min } \\
& =H_{l}^{(-)}(k r)-S_{l} H_{l}^{(+)}(k r), \quad r \rightarrow \infty
\end{aligned}
$$

Here, $H_{l}^{( \pm)}$are the Coulomb wave functions, and $k$ and $k_{l}(r)$ are defined as $k=\sqrt{2 \mu E / \hbar^{2}}$ and

$$
k_{l}(r)=\sqrt{\frac{2 \mu}{\hbar^{2}}\left(E-V_{0}(r)-\frac{l(l+1) \hbar^{2}}{2 \mu r^{2}}\right)},
$$


respectively. $r_{\min }$ is the radius at which the incoming wave boundary condition is imposed and is taken somewhere inside the Coulomb barrier [2, 9]. From the $S$ matrix, $S_{l}$, or the transmission coefficient, $T_{l}$, so obtained, fusion cross sections are evaluated as,

$$
\sigma_{\text {fus }}(E)=\frac{\pi}{k^{2}} \sum_{l=0}^{\infty}(2 l+1) P_{l}(E),
$$

with $P_{l}(E)=1-\left|S_{l}\right|^{2}=\left|T_{l}\right|^{2}$.

In order to derive an analytic expression for fusion cross sections, Wong has first approximated the Coulomb barrier with an inverted parabola, that is,

$$
V_{0}(r) \sim V_{B}-\frac{1}{2} \mu \Omega^{2}\left(r-R_{B}\right)^{2} .
$$

In the original Wong formula, the barrier position, $R_{B}$, and the barrier curvature, $\hbar \Omega$, are assumed to be independent of $l$ and are evaluated for the $s$-wave. The effective potential for the $l$-th partial wave then reads,

$$
\begin{aligned}
V_{0}(r)+\frac{l(l+1) \hbar^{2}}{2 \mu r^{2}} \sim & V_{B}+\frac{l(l+1) \hbar^{2}}{2 \mu R_{B}{ }^{2}} \\
& -\frac{1}{2} \mu \Omega^{2}\left(r-R_{B}\right)^{2} .
\end{aligned}
$$

Using the Hill-Wheeler formula [10], the penetration probability, $P_{l}(E)$, is calculated as,

$$
P_{l}(E)=\frac{1}{1+\exp \left[\frac{2 \pi}{\hbar \Omega}\left(V_{B}+\frac{l(l+1) \hbar^{2}}{2 \mu R_{B}^{2}}-E\right)\right]} .
$$

Replacing the summation in Eq. (5) by the integral, that is,

$$
\frac{\pi}{k^{2}} \sum_{l=0}^{\infty}(2 l+1) P_{l}(E) \rightarrow \frac{\pi}{k^{2}} \int_{0}^{\infty} d l(2 l+1) P_{l}(E),
$$

one finally obtains the well-known Wong formula given by

$$
\sigma_{\text {fus }}(E)=\frac{\hbar \Omega}{2 E} R_{B}^{2} \ln \left[1+\exp \left(\frac{2 \pi}{\hbar \Omega}\left(E-V_{B}\right)\right)\right] .
$$

Notice that at energies well above the Coulomb barrier, i.e., $E-V_{B} \gg \hbar \Omega / 2 \pi$, the Wong formula leads to the classical fusion cross section,

$$
\sigma_{\text {fus }}(E) \sim \pi R_{B}^{2}\left(1-\frac{V_{B}}{E}\right) .
$$

See Appendix B in Ref. [2] for the performance of the parabolic approximation and the Wong formula for the ${ }^{16} \mathrm{O}+{ }^{144} \mathrm{Sm}$ system.

Figure 1 compares fusion cross sections with the Wong formula (the dashed line) to those obtained by numerically solving the Schrödinger equation (the solid line), for the ${ }^{12} \mathrm{C}+{ }^{16} \mathrm{O},{ }^{40} \mathrm{Ca},{ }^{154} \mathrm{Sm}$ and ${ }^{238} \mathrm{U}$ systems. To this
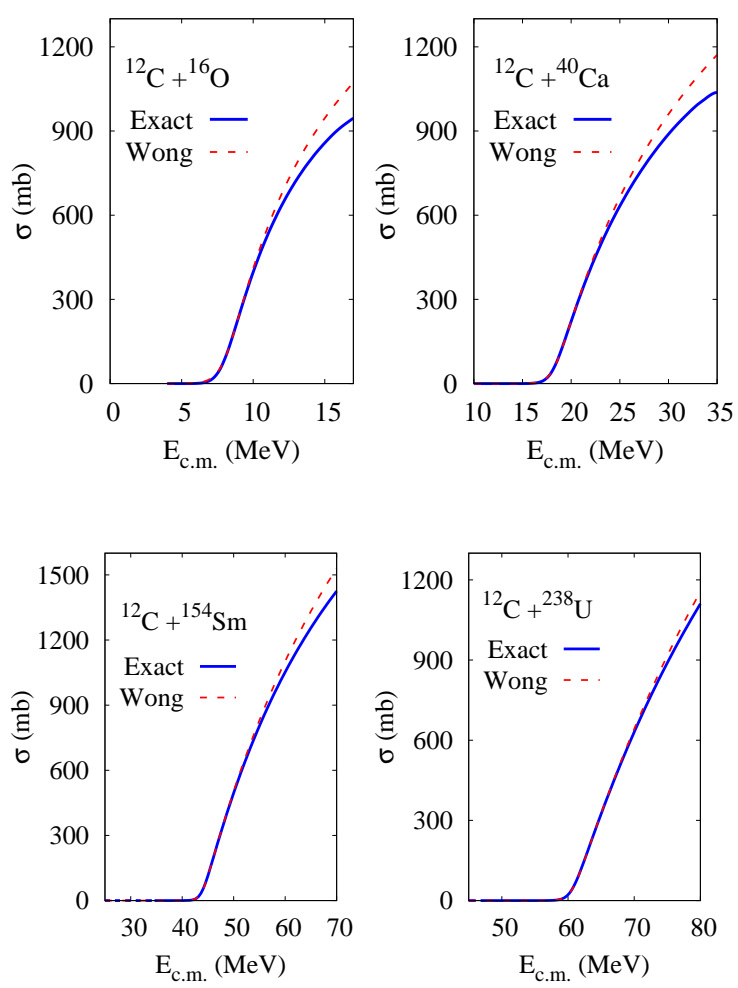

FIG. 1: Comparisons between the exact (the solid line) and approximate (the dashed line) fusion cross sections for four selected systems. The approximate solutions are obtained with the Wong formula. The Akyüz-Winther potential is employed for the internuclear potential.

end, we employ the Akyüz-Winther potential [11] for a nuclear part of the nucleus-nucleus potential. As can be seen in the figure, the Wong formula tends to overestimate fusion cross sections, although the agreement with the exact results considerably improves as the mass number of the target increases [8] (see also Ref. [12]).

\section{SYSTEMATICS}

In order to discuss more systematically the performance of the Wong formula, in this section we vary the target nucleus from $\mathrm{C}$ to $\mathrm{U}$ for a fixed projectile nucleus. For each target nuclide, we choose the isotope which has the largest natural abundance. As the projectile nucleus, we choose ${ }^{4} \mathrm{He},{ }^{6} \mathrm{Li},{ }^{12} \mathrm{C},{ }^{16} \mathrm{O}$, and ${ }^{20} \mathrm{Ne}$. In order to compare the deviation of the Wong formula in different systems, we introduce the following quantity:

$$
\Delta \sigma \equiv \frac{\int_{E_{\min }}^{E_{\max }}\left|\sigma_{\text {exact }}(E)-\sigma_{\text {Wong }}(E)\right| d E}{\int_{E_{\min }}^{E_{\max }} \sigma_{\text {exact }}(E) d E} .
$$

We choose the minimum and the maximum energies for the integration to be $E_{\min }=0.9 V_{B}$ and $E_{\max }=1.1 V_{B}$, respectively. 




FIG. 2: The deviation from the exact results, defined by Eq. (12), of the fusion cross sections obtained with the Wong formula. This quantity is plotted as a function of the mass number of the target nucleus for five projectile nuclei indicated in the figure.

Figure 2 shows the calculated results as a function of the mass number $A$ of the target nucleus. We again use the Akyüz-Winther potential for each system. One can see that the deviation, $\Delta \sigma$, decreases as a function of the mass number of the target nucleus. For the ${ }^{12} \mathrm{C},{ }^{16} \mathrm{O}$, and ${ }^{20} \mathrm{Ne}$ projectiles, the deviation almost saturates at around $A \sim 50$, that corresponds to $Z \sim 20$. On the other hand, for the lighter projectiles, the decrease of $\Delta \sigma$ is much slower and especially for ${ }^{4} \mathrm{He}$ the deviation is relatively large even for a heavy target. This implies that the original Wong formula is not applicable for light projectiles such as ${ }^{4} \mathrm{He}$, and the generalization proposed in Ref. [8] is important. For heavier projectile nuclei, as we have noted, the original Wong formula works well as long as the mass number of the target nucleus is around $A \sim 50$ or larger.

In order to see the energy dependence of the deviation of the Wong formula, we next define a quantity $\Delta(E)$

$$
\Delta(E) \equiv \frac{\sigma_{\text {exact }}(E)-\sigma_{\text {Wong }}(E)}{\sigma_{\text {exact }}(E)},
$$

which indicates the degree of deviation at a given energy, $E$. Fig. 3 shows the quantity $\Delta(E)$ for the ${ }^{6} \mathrm{Li}$ projectile on several target nuclei from $\mathrm{O}$ to $\mathrm{U}$, as a function of energy relative to the barrier height for each system. Except for the energy region just above the barrier, $\Delta(E)$ is negative, that is, the Wong formula overestimates fusion cross sections. Furthermore, the slope of $\Delta(E)$ is much steeper for the lighter targets compared to that for the heavier targets, that is consistent with the finding shown in Fig. 2 ,

At energies slightly above the barrier, the deviation $\Delta(E)$ is positive, thus the Wong formula underestimates fusion cross sections. This is due to the fact that the parabolic approximation underestimates the width of the Coulomb barrier because of the asymmetric shape of the



FIG. 3: The deviation of the Wong formula given by Eq. (13) for ${ }^{6} \mathrm{Li}$ induced fusion reactions as a function of the incident energy relative to the barrier height for each system. The target nucleus is ${ }^{238} \mathrm{U},{ }^{152} \mathrm{Sm},{ }^{74} \mathrm{Ge},{ }^{40} \mathrm{Ar},{ }^{28} \mathrm{Si},{ }^{20} \mathrm{Ne}$, and ${ }^{16} \mathrm{O}$ in the decreasing order at $E-V_{B}=4 \mathrm{MeV}$.

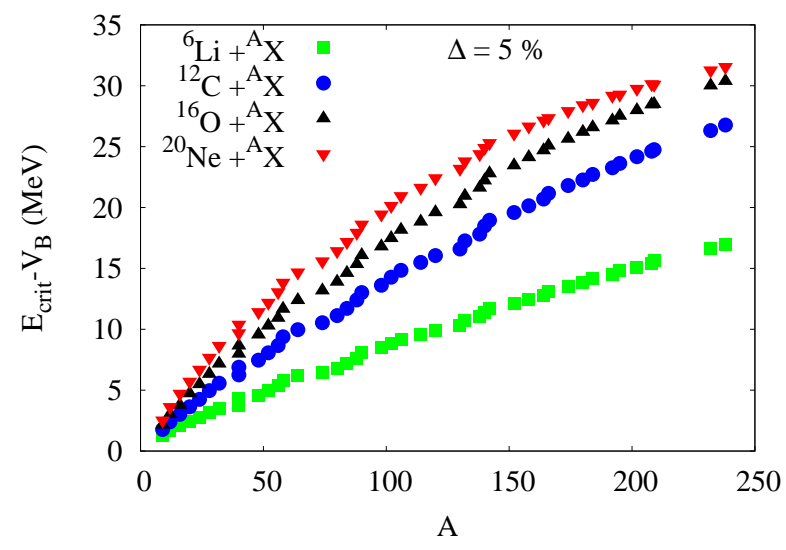

FIG. 4: The critical energy, $E_{\text {crit }}$, at which the deviation defined by Eq. (13) exceeds $5 \%$, measured relative to the barrier height for each system as a function of the mass number of the target nucleus.

Coulomb barrier caused by the long range Coulomb interaction (see Fig. 15 in Ref. [2]). This leads to a smaller tunneling probability at energies above the barrier, thus reducing fusion cross sections. At higher energies, on the other hand, the tunneling is not important, and the $l$-dependence of the barrier position $R_{\mathrm{B}}$ comes into a play to reduce fusion cross sections from the approximate cross sections [8].

Let us next define the critical energy $E_{\text {crit }}$ as the energy at which the absolute value of the deviation, $|\Delta(E)|$, exceeds a certain value. We arbitrarily choose it to be $5 \%$. Fig. [4shows the critical energy with respect to the barrier height for each system as a function of the mass number of the target nucleus. For the projectile, we choose ${ }^{6} \mathrm{Li}$, ${ }^{12} \mathrm{C},{ }^{16} \mathrm{O}$, and ${ }^{20} \mathrm{Ne}$. The figure shows that for light systems the critical energy is reached already at an energy 
slightly above the barrier, while one has to go up to a higher energy for heavy systems. For instance, the critical energy is $3.02 \mathrm{MeV}$ higher than the barrier for the ${ }^{12} \mathrm{C}+{ }^{16} \mathrm{O}$ system whereas it is $30.37 \mathrm{MeV}$ higher than the barrier for the ${ }^{12} \mathrm{O}+{ }^{238} \mathrm{U}$ system. We notice that this is consistent with what we have shown in Fig. 1.

\section{SUMMARY}

We have carried out a comparative study on the applicability of the Wong formula for single-channel fusion cross sections. To this end, we have compared the fusion cross sections obtained with the Wong formula to the ex- act results from light to heavy systems. We have shown that the Wong formula leads to reasonable results when the target nucleus is in the $A \sim 50$ region or heavier for the projectiles of ${ }^{6} \mathrm{Li},{ }^{12} \mathrm{C},{ }^{16} \mathrm{O}$, and ${ }^{20} \mathrm{Ne}$, while the deviation is still large even for a heavy target in systems with ${ }^{4} \mathrm{He}$ projectile. We have also investigated the energy dependence of the deviation, and have shown that the deviation quickly becomes large for light systems as the energy increases while a high incident energy is required for heavy systems before the deviation becomes significant. These conclusions indicate that a care must be taken in using the Wong formula in light systems, for which the generalization discussed in Ref. [8] becomes essential.
[1] A.B. Balantekin and N. Takigawa, Rev. Mod. Phys. 70, 77 (1998).

[2] K. Hagino and N. Takigawa, Prog. Theor. Phys. 128, 1001 (2012).

[3] M. Dasgupta, D.J. Hinde, N. Rowley, and A.M. Stefanini, Annu. Rev. Nucl. Part. Sci. 48, 401 (1998).

[4] B.B. Back, H. Esbensen, C.L. Jiang, and K.E. Rehm, Rev. Mod. Phys. 86, 317 (2014).

[5] C.Y. Wong, Phys. Rev. Lett. 31, 766 (1973).

[6] K. Hagino, Phys. Rev. C93, 061601(R) (2016).

[7] N. Poffé, N. Rowley and R. Lindsay, Nucl. Phys. A410, 498 (1983).
[8] N. Rowley and K. Hagino, Phys. Rev. C 91, 044617 (2015).

[9] K. Hagino, N. Rowley, A.T. Kruppa, Comp. Phys. Comm. 123, 143 (1999).

[10] D.L. Hill and J.A. Wheeler, Phys. Rev. 80, 1102 (1953).

[11] O. Akyüz and A. Winther, Proc. Int. School of Physics Enrico Fermi, Course LXXVII, edited by R. A. Broglia, R. A. Ricci, and C. H. Dasso (Noah-Holland, Amsterdam, 1981), p. 492.

[12] A.J. Toubiana, L.F. Canto, and M.S. Hussein, Euro. Phys. J. 53, 34 (2017). 